\title{
Multi-spacecraft observations of broadband waves near the lower hybrid frequency at the Earthward edge of the magnetopause
}

\author{
M. André ${ }^{1}$, R. Behlke ${ }^{1}$, J.-E. Wahlund ${ }^{1}$, A. Vaivads ${ }^{1}$, A.-I. Eriksson ${ }^{1}$, A. Tjulin ${ }^{1}$, T. D. Carozzi ${ }^{1}$, C. Cully ${ }^{1,2}$, G. \\ Gustafsson $^{1}$, D. Sundkvist ${ }^{1}$, Y. Khotyaintsev ${ }^{1}$, N. Cornilleau-Wehrlin ${ }^{3}$, L. Rezeau ${ }^{3}$, M. Maksimovic ${ }^{4}$, E. Lucek ${ }^{5}$, A.

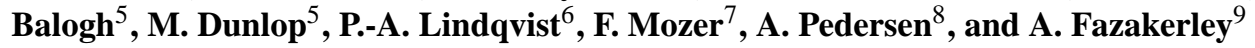 \\ ${ }^{1}$ Swedish Institute of Space Physics, Uppsala division, Sweden \\ ${ }^{2}$ University of Calgary, Canada \\ ${ }^{3}$ CETP, Vélizy, France \\ ${ }^{4}$ DESPA/CNRS, Observatoire de Paris, Meudon, France \\ ${ }^{5}$ The Blackett Laboratory, Imperial College, London, UK \\ ${ }^{6}$ Royal Institute of Technology, Stockholm, Sweden \\ ${ }^{7}$ Space Science Laboratory, University of California at Berkeley, Berkeley, USA \\ ${ }^{8}$ Department of Physics, University of Oslo, Norway \\ ${ }^{9}$ Mullard Space Science Laboratory, University College London, London, UK
}

Received: 13 April 2001 - Revised: 21 June 2001 - Accepted: 23 June 2001

\begin{abstract}
Broadband waves around the lower hybrid frequency (around $10 \mathrm{~Hz}$ ) near the magnetopause are studied, using the four Cluster satellites. These waves are common at the Earthward edge of the boundary layer, consistent with earlier observations, and can have amplitudes at least up to $5 \mathrm{mV} / \mathrm{m}$. These waves are similar on all four Cluster satellites, i.e. they are likely to be distributed over large areas of the boundary. The strongest electric fields occur during a few seconds, i.e. over distances of a few hundred $\mathrm{km}$ in the frame of the moving magnetopause, a scale length comparable to the ion gyroradius. The strongest magnetic oscillations in the same frequency range are typically found in the boundary layer, and across the magnetopause. During an event studied in detail, the magnetopause velocity is consistent with a large-scale depression wave, i.e. an inward bulge of magnetosheath plasma, moving tailward along the nominal magnetopause boundary. Preliminary investigations indicate that a rather flat front side of the large-scale wave is associated with a rather static small-scale electric field, while a more turbulent backside of the large-scale wave is associated with small-scale time varying electric field wave packets.
\end{abstract}

Key words. Magnetospheric physics (magnetopause, cusp, and boundary layers) - Space plasma physics (waves and instabilities)

\section{Introduction}

The magnetopause is a complex boundary between the Earth's magnetosphere and the shocked solar wind. The solar wind-magnetosphere interaction never ceases, and in a

Correspondence to: M. André (mats.andre@irfu.se) global perspective, particles, energy and momentum are essentially constantly transferred across the magnetopause to the magnetoshere. Waves at frequencies from well below the proton gyrofrequency (of the order of $1 \mathrm{~Hz}$ ) up to higher frequencies may be important for particle diffusion across the magnetopause, and for the onset of reconnection. We present here observations of electric and magnetic field variations up to $10 \mathrm{~Hz}$ and higher in the spacecraft frame of reference, obtained by the four Cluster satellites during multiple crossings of the duskside magnetopause. Our investigation includes data from the first double probe electric field experiment that at high sampling rates, 25 samples/s or higher, routinely provides observations from the outer boundaries of the magnetosphere.

The large-scale shape and location of the magnetopause have been mapped by earlier spacecraft, and this boundary is structured on many length scales (Russell, 1995; Fairfield, 1995). In addition, waves covering a broad frequency range are usually present near the magnetopause, as observed by several single spacecraft (Holzer et al., 1966; Gurnett et al., 1979; Blecki et al., 1988; Labelle and Treumann, 1988; Tsurutani et al., 1989; Thorne and Tsurutani, 1990; Anderson, 1995; Lucek et al., 2001) and there are also occasional observations obtained simultaneously by two satellites (Rezeau et al., 1993). The importance of these waves for diffusion and for the onset of reconnection is discussed in reviews by Labelle and Treumann (1988), Sibeck et al. (1999) and Rezeau and Belmont (2001).

In the following, we present observations from the four Cluster satellites of electric and magnetic fields and waves, and plasma density from an orbit where the spacecraft experienced multiple crossings of the duskside magnetopause. We investigate the details of the electric field at the boundary 


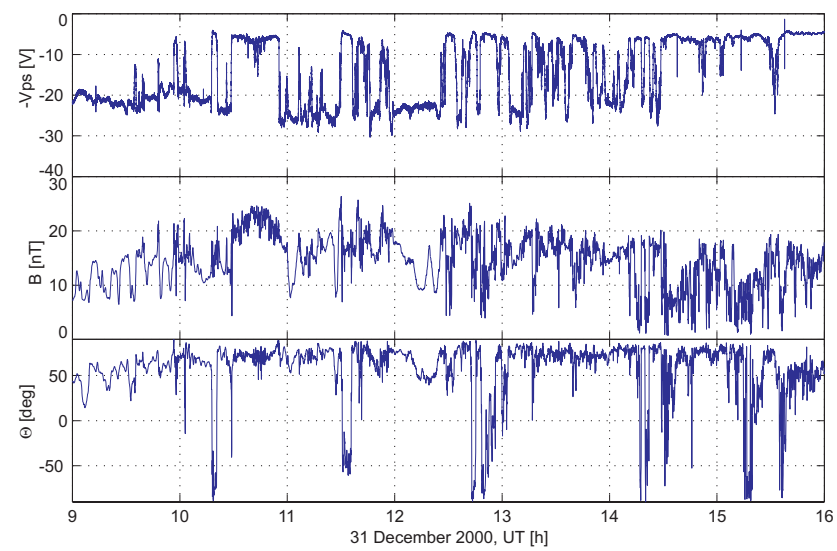

Fig. 1. Observations by Cluster satellite 4 on 31 December 2000. The upper panel shows the potential $v_{p s}$. This parameter is positively correlated with the density, i.e. a high $v_{p s}$ corresponds to a high density. A $v_{p s}$ of $-20,-10$ and $-5 \mathrm{~V}$ corresponds to about 0.8 , 2 and 20 particles $\mathrm{cm}^{-3}$. The middle panel displays the magnetic field from FGM, and the bottom panel shows the elevation angle of this field in GSE coordinates (so that $90^{\circ}$ corresponds to a strictly northward field).

of a large-scale magnetopause wave, a tailward moving inward bulge of magnetosheath plasma. Preliminary results indicate that on the front side of the large-scale wave, the electric fields are rather static in the magnetopause frame, while the electric fields on the more turbulent "backside" of the large-scale wave consist of time varying electric field wave packets.

\section{Cluster instruments}

Cluster is a unique four satellite mission designed to investigate the Earth's magnetosphere and its boundaries in detail (Escoubet et al., 1997). In this study, we use data from the Electric Field and Wave (EFW) instrument with two pairs of probes on wire booms in the spin plane of each satellite. Each pair has a probe-to-probe separation of $88 \mathrm{~m}$ (Gustafsson et al., 1997, 2001). For the event presented here, a sampling rate of 25 samples/s with an anti-aliasing filter at 10 $\mathrm{Hz}$ was used for the electric field. In addition, the potential of the probes with respect to the spacecraft was sampled at 5 samples/s, and this measurement can be used to estimate the plasma density (Pedersen, 2001). We also use data from the STAFF search coil magnetometers (Cornilleau-Wehrlin et al., 1997) and the FGM fluxgate magnetometers (Balogh et al., 1997).

\section{Observations}

On 31 December 2000, the Cluster satellites crossed the high-latitude duskward magnetopause several times. The upper panel of Fig. 1 shows the probe-to-spacecraft potential $v_{p s}$ (EFW on satellite 4) obtained by taking the average of the difference between the probe and the satellite potential for all four probes. This difference is often referred to as the negative of the spacecraft potential. The potential $v_{p s}$ is positively correlated with the density, i.e. a high $v_{p s}$ corresponds to a high density. During the 7 hours displayed in the figure, the satellites moved from $(3,11,9) \mathrm{R}_{\mathrm{e}}$ in GSE coordinates to $(6,15,7) \mathrm{R}_{\mathrm{e}}$ with spacecraft separations of typically a few hundred kilometres to a thousand kilometers. In Fig. 1, a $v_{p s}$ of $-20,-10$ and $-5 \mathrm{~V}$ corresponds to about $0.8,2$ and 20 particles $\mathrm{cm}^{-3}$. The middle panel of Fig. 1 shows the magnitude of the magnetic field observed by FGM, while the bottom panel shows the elevation angle of the magnetic field in GSE coordinates (so that $+90^{\circ}$ corresponds to a northward field). In the upper panel of Fig. 1, a low $v_{p s}$ corresponds to the magnetosphere, with several increases to around $-10 \mathrm{~V}$ indicating entries into a boundary layer, while increases to around -5 may represent crossings of the magnetopause into the magnetosheath. An increase in $v_{p s}$, together with a clear decrease in the elevation of the magnetic field, such as around 10:20 and 11:30 UT, indicate clear excursions into the magnetosheath. Since the satellite speed of the order of $1 \mathrm{~km} / \mathrm{s}$ is much lower than the plasma speed, a more appropriate description may be that the magnetopause motion brings the satellite into the magnetosheath. In comparison to a nominal model magnetopause, the satellites are always outside this boundary at distances from 0.5 $\mathrm{R}_{\mathrm{e}}$ (beginning of the period) to $2 \mathrm{R}_{\mathrm{e}}$ (end of the period). The multiple crossings in Fig. 1 indicate significant motion of the magnetopause, although the WIND spacecraft (at a distance of about $250 \mathrm{R}_{\mathrm{e}}$ from Earth) observed a rather steady solar wind with a pressure of about $1 \mathrm{nPa}$. As discussed below, at least some of the magnetopause crossings correspond to large-scale surface waves rather than just radial motion of the magnetopause.

Figure 2 shows 22 minutes of data, starting at 10:00 UT, on 31 December 2000. Panel (a) shows $v_{p s}$ as in Fig. 1, i.e. an indication of the density, plotted for all four Cluster spacecraft. The satellites encounter a boundary layer and possibly the magnetosheath for about a minute after 10:02 UT; there is a significant density perturbation just after 10:05 UT, and the spacecraft then enter the magnetosheath between about 10:17:30 UT and 10:21:00 UT. Panels (b-e) show EFW $E_{y}$ (in GSE coordinates) electric field data from satellites 1 to 4 at frequencies up to $10 \mathrm{~Hz}$. It is clear that broadband (in the satellite frame of reference) waves are seen on all spacecraft at density gradients, and sometimes in nearby low density magnetospheric regions. (The signal up to around $1 \mathrm{~Hz}$ in this figure is primarily caused by an artificial non-linear coupling between the plasma and the probes. In the magnetosheath, such signals can be seen up to about $3 \mathrm{~Hz}$.) Panel (f) displays the electric field between 10 and $50 \mathrm{~Hz}$, and panel (g) shows the magnetic field up to $10 \mathrm{~Hz}$; both panels use data from the STAFF instrument on satellite 4 . The proton gyrofrequency is about $0.3 \mathrm{~Hz}$, and the lower hybrid frequency is approximately $10 \mathrm{~Hz}$. Panel (f) shows that the broadband waves observed by EFW exist also at higher frequencies. By comparing panels e and $\mathrm{g}$, we find that the strong electric and 


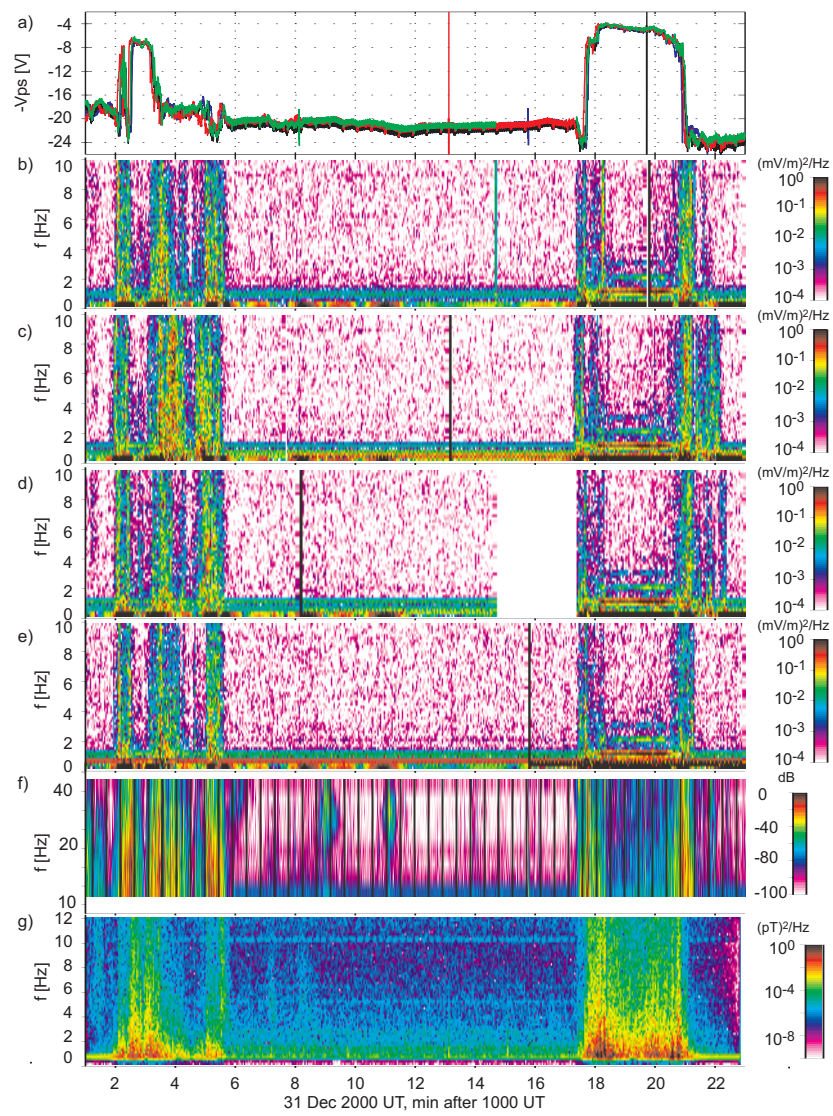

Fig. 2. Cluster observations from 31 December 2000. Panel (a) shows the potential $v_{p s}$ from EFW (satellites 1-4 correspond to black, red, green and blue, respectively). A $v_{p s}$ of $-20,-10$ and -5 $\mathrm{V}$ corresponds to about $0.8,2$ and 20 particles $\mathrm{cm}^{-3}$. Panels (b-e) display EFW $E_{y}$ electric field up to $10 \mathrm{~Hz}$. (The signal from around the satellite spin frequency $(0.25 \mathrm{~Hz})$ to $1 \mathrm{~Hz}$ is primarily artificial, see text). Panels (f) and (g) show satellite 4 STAFF electric fields $(10-50 \mathrm{~Hz})$ and the total magnetic (up to $10 \mathrm{~Hz}$ ) fields, respectively. Note the broadband electric emissions at the potential gradients, and the magnetic emissions in regions of high $v_{p s}$.

magnetic oscillations at a few $\mathrm{Hz}$ are nearly anti-correlated; the former is confined to density gradients at well at the low density magnetosphere, and the latter is primarily occurring in the boundary layer and the magnetosheath with higher densities.

Figure 3 displays the latter part of the event in Fig. 2 in higher time resolution. It is again clear that the strongest electric field emissions are detected near density gradients, and also that such waves may occur elsewhere in the low density magnetosphere. (As in Fig. 3, some signals in the magnetosheath up to about $3 \mathrm{~Hz}$ are due to artificial non-linear coupling.) Note that satellite 2 moved into a boundary layer around 10:17:35 UT, a few seconds before the other spacecraft, and that the broadband emission shown in panel (c) accordingly occurs a few seconds before the emissions on the other spacecraft. Panels (h) and (i) of Fig. 3 show the magnitude of the magnetic field and the elevation angle on satellite 4 (from FGM). The decrease (increase) in the elevation an-

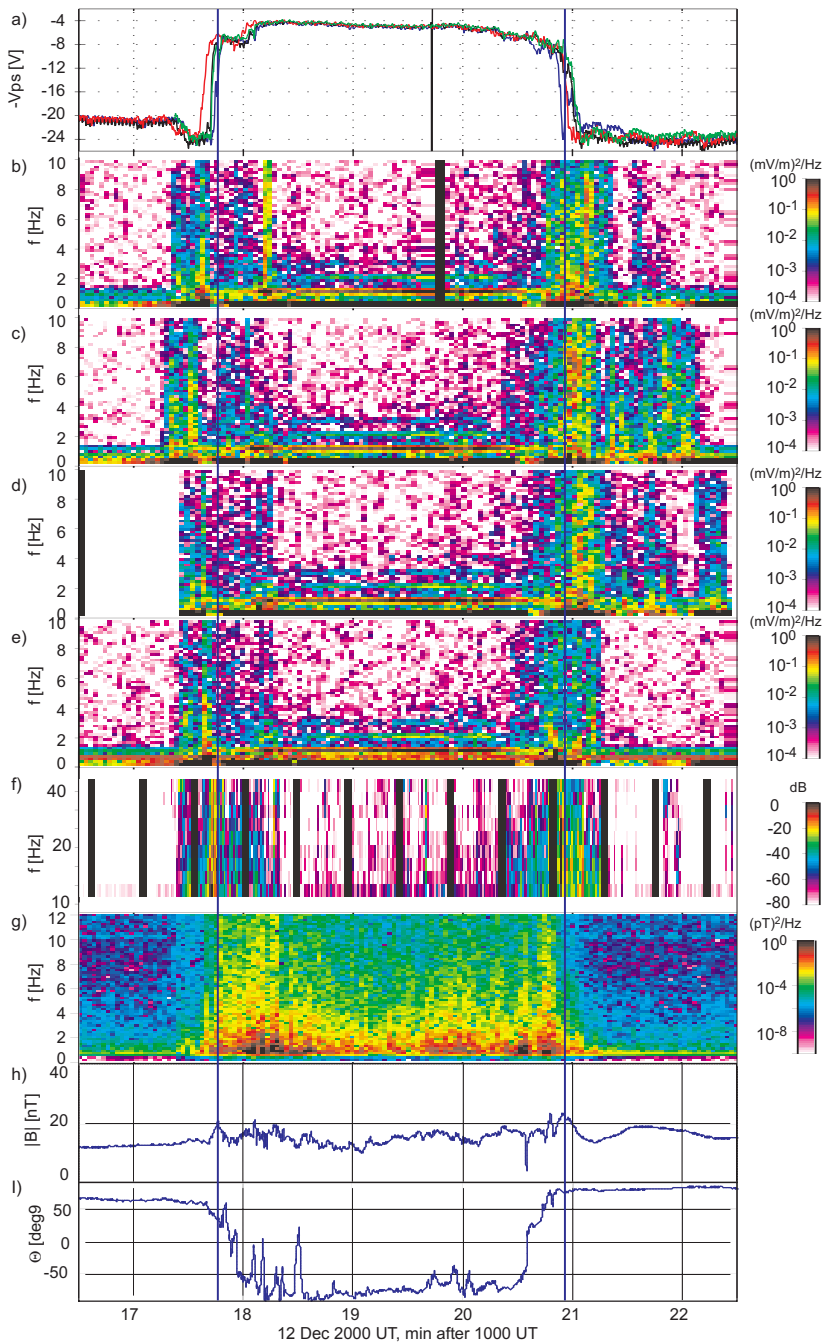

Fig. 3. Cluster observations from 31 December 2000. This display corresponds to the latter part of Fig. 2, and the upper seven panels are similar to that figure. (Note the data gap during the first minute in panel (d). Panels (h) and (i) show the magnitude of the magnetic field from FGM on satellite 4, and the elevation angle of this field (so that $90^{\circ}$ corresponds to a strictly northward field), respectively.

gle near 10:18:00 UT (10:20:30 UT) is consistent with the spacecraft crossing the magnetopause and entering (leaving) the magnetosheath. Note that the sharp increase (decrease) in $v_{p s}$ occurs a few seconds before (after) the sudden change in the elevation angle of the magnetic field. Starting at the magnetospheric side, these data are consistent with first, a density increase when moving into a boundary layer, and then a change in the magnetic field direction, which is consistent with a current sheet when moving into the magnetosheath. (The decrease in $v_{p s}$ to $-24 \mathrm{~V}$ just after 10:17:30 UT may be due to not only low density but also to low electron temperature.) Panel (h) shows magnetic fluctuations consistent with the low frequency part of panel $(\mathrm{g})$. Considering the waves at frequencies up to $10 \mathrm{~Hz}$, at least during this event, the strongest broadband wave electric fields tend occur at the Earthward edge of the boundary layer, while the strongest 

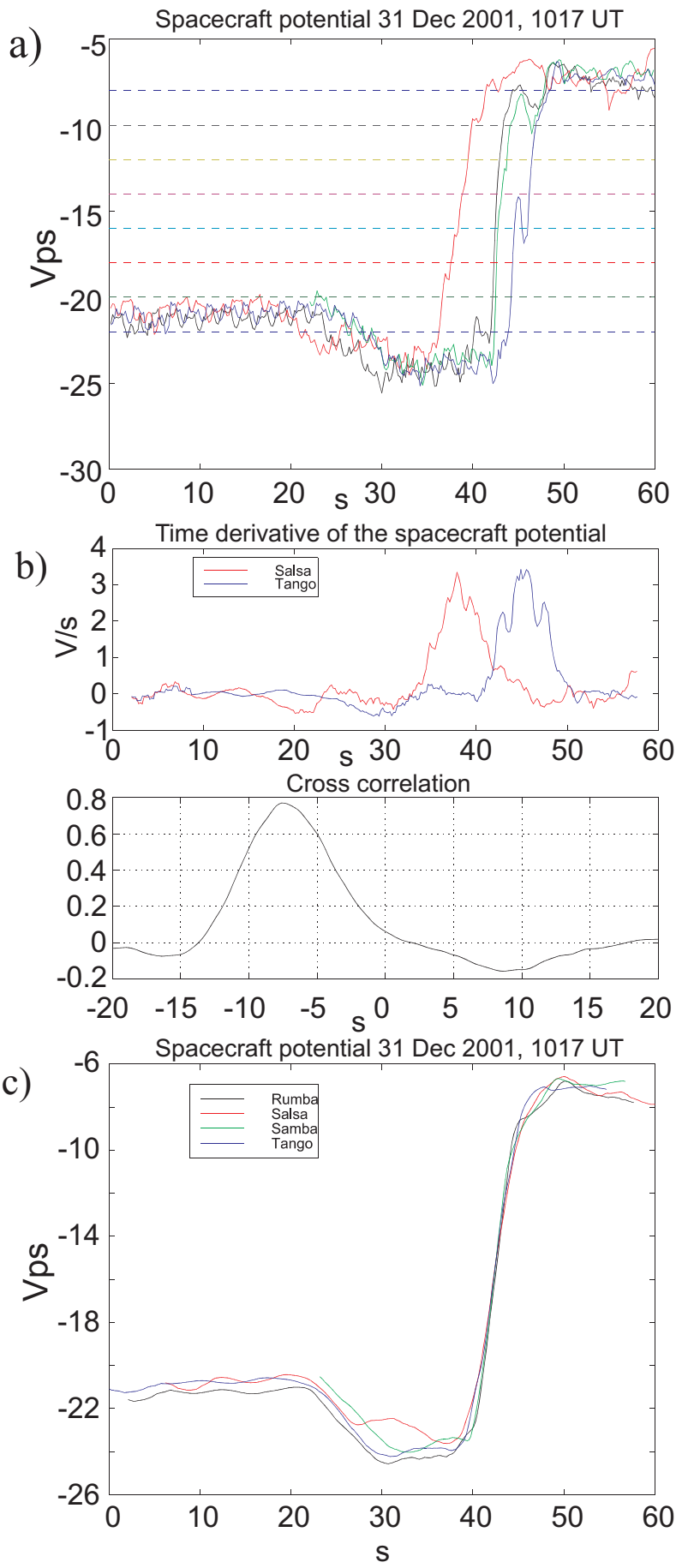

Fig. 4. (a) The parameter $v_{p s}$ for all four satellites, with a few specific potentials indicated, (b) the derivative of this potential for two satellites and the corresponding cross-correlation, (c) the low pass filtered potentials shifted in time to overlap. In this lower panel $10 \mathrm{~s}$ correspond to about $1000 \mathrm{~km}$.

magnetic oscillations appear in the boundary layer and also across the magnetopause into the magnetosheath.

The magnetopause and the associated electric and magnetic oscillations are moving across the Cluster satellites. The velocity of the magnetopause can be estimated by vari- ous methods. One method is to consider one specific value of $v_{p s}$ and note the times when each of the four spacecraft reaches that level (Fig. 4a).

With this information and the knowledge of the spacecraft positions, the velocity of the density structure can be calculated. This is done under two assumptions: that the magnetopause locally is flat, and that the velocity is constant and normal to the magnetopause. Another way is similar to the method used by Bale et al. (2001). By computing crosscorrelations of the derivatives of the time-series of $v_{p s}$, it is possible to find the time delay between the different spacecraft passing the structure (Fig. 4b). Using the same assumptions as above, the velocity can be calculated. By using the derivative in the cross-correlation rather than $v_{p s}$, the transition into the structure is emphasized. Having estimated the magnetopause velocity, the potential $v_{p s}$ from different spacecraft can now be shifted in time and we find that they overlap (Fig. 4c). A certain time interval in the satellite frame now corresponds to a certain length interval in the magnetopause frame. The methods described above give similar velocities, around $v_{n}=(-100,-10,-55) \mathrm{km} / \mathrm{s}$. The speed is about $110 \mathrm{~km} / \mathrm{s}$, with a variation between estimates of less than $10 \mathrm{~km} / \mathrm{s}$, and the variation in direction of less than $15^{\circ}$. Here we note that preliminary analysis of the FGM magnetometer data, including minimum variance analysis, is in reasonable agreement with these observations. Future studies should include more minimum variance analysis of electric and magnetic fields to estimate the normal of the magnetopause at each satellite, and to test the assumption of a flat magnetopause. Figure 5 shows a sketch of the location of the satellites, and the magnetopause and its velocity.

Some details of the moving magnetopause and boundary layer are considered in Fig. 6a. A time series of the $E_{y}$ electric field component from satellite 2, near the boundary crossing leaving the magnetosphere around 10:17:30 UT, is shown in the upper panel of Fig. 6a. (Compensation for slight differences between probes have been used in this figure. In addition, some effects of the wake caused by the satellite body in the magnetosheath has been removed by filtering.) The electric field has one single major negative peak at 10:17:35 UT. This peak occurs just before the increase in $v_{p s}$. This single peak gives a significant contribution to the broadband spectrum in panel (c) of Fig. 3. Other electric field oscillations are concentrated in the region of lowest $v_{p s}$, from 10:17:20 UT to 10:17:35 UT. As mentioned before, this low $v_{p s}$ may be due not only to low density, but also to low electron temperature. The lower panel of Fig. 6a shows the electric field in the frequency domain, using a wavelet transform. This display gives a high time resolution at higher frequencies, and clearly shows the broadband character of the single electric field peak in frequency space, and also the location of the electric field emissions to the region of low $v_{p s}$.

A time series of $E_{y}$ from satellite 2, near the boundary crossing entering the magnetosphere around 10:21:00 UT, is displayed in the upper panel of Fig. 6b. The electric field is found in small regions, each including a few oscillations at a few Hz. The oscillations occur just after the sharp decrease 


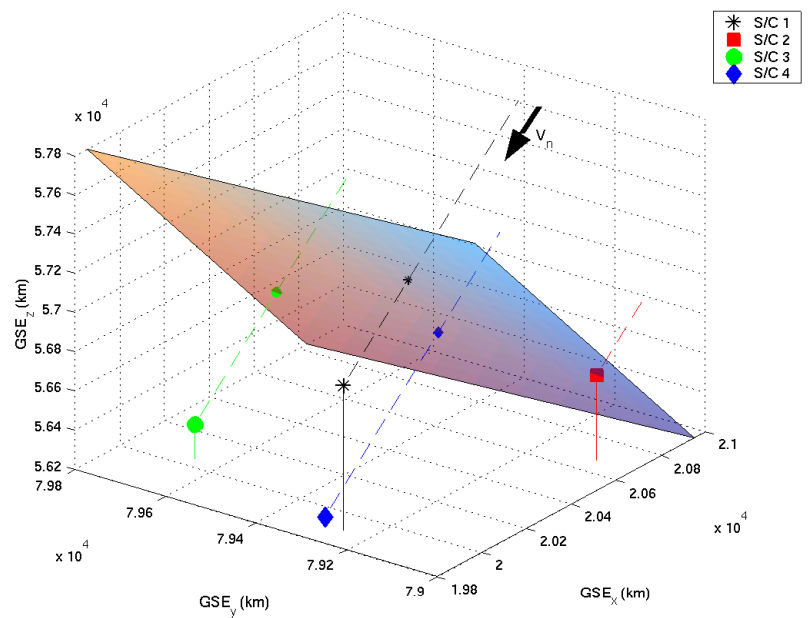

Fig. 5. A sketch of the location of the Cluster satellites and the orientation of the magnetopause during the magnetopause crossing on 31 December 2000, near 10:17 UT. Satellites 1 to 4 are indicated by black, red green and blue, respectively.

in $v_{p s}$. The lower panel of Fig. $6 \mathrm{~b}$ shows this electric field in the frequency domain. Similar to the example above, the strongest electric fields are found just after the decrease in $v_{p s}$. In contrast to the example above, here we find a few small "wavepackets", each containing a few ocsillations at a few $\mathrm{Hz}$.

An important point is how much of the observed electric field variations are true time variations in the reference frame of the magnetopause, and how much is due to a Doppler shift in the satellite frame caused by the motion of the magnetopause. To investigate this, it is useful to plot the electric field time series from each satellite in a common reference frame, using the magnetopause velocity estimated above. In Fig. 7a, the $E_{y}$ component from all four satellites near the outbound crossing at around 10:18 UT is plotted as a function of time. The red line corresponding to satellite 2 is the same electric field as in Fig. 6a. The first major negative peak of the electric field is indicated for each spacecraft. As for satellite 2, each indicated peak occurs near (within about a second) the start of the sharp rise in $v_{p s}$. In Fig. 7b, the same time series are shifted using the magnetopause velocity obtained from changes in the $v_{p s}$ parameter observed on each satellite (see Fig. 4). Here, the indicated peaks nearly overlap, occurring within less than one second. In addition, at times before 10:17:38 UT and after 10:17:42 UT in Fig. 7b, the fluctuations are rather small.

An electric field with a component of a few $\mathrm{mV} / \mathrm{m}$ in the negative $y$ direction exists on all four spacecraft. The time series from the different satellites are far from identical, and it may be argued that other peaks should also be indicated as major disturbances. However, we emphasize that we use one parameter, $v_{p s}$, obtained on all four satellites to estimate the magnetopause velocity. This velocity is then used to study another parameter, the electric field, and to dicover that similar structures overlap when plotted in the moving magne-

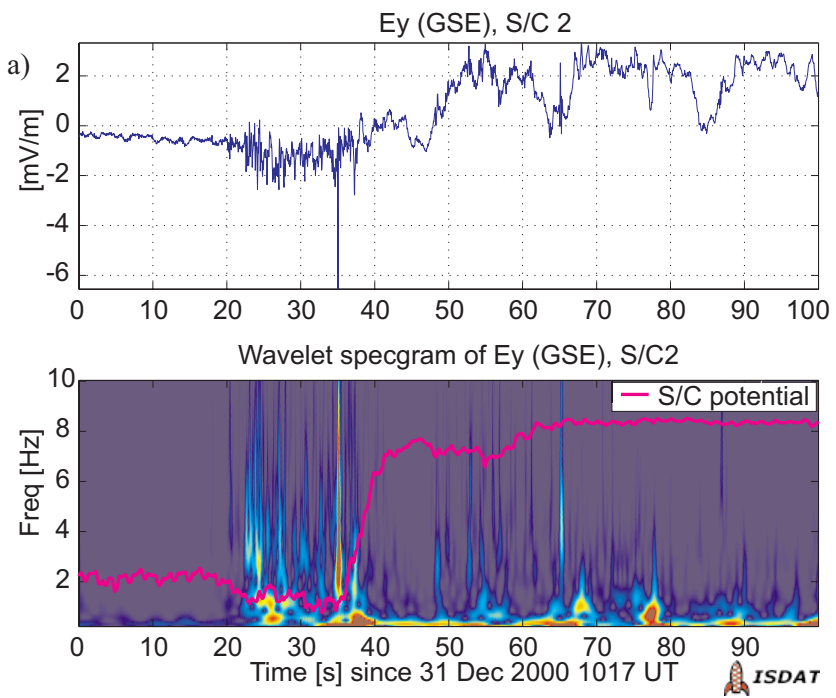

Ey (GSE), S/C 2
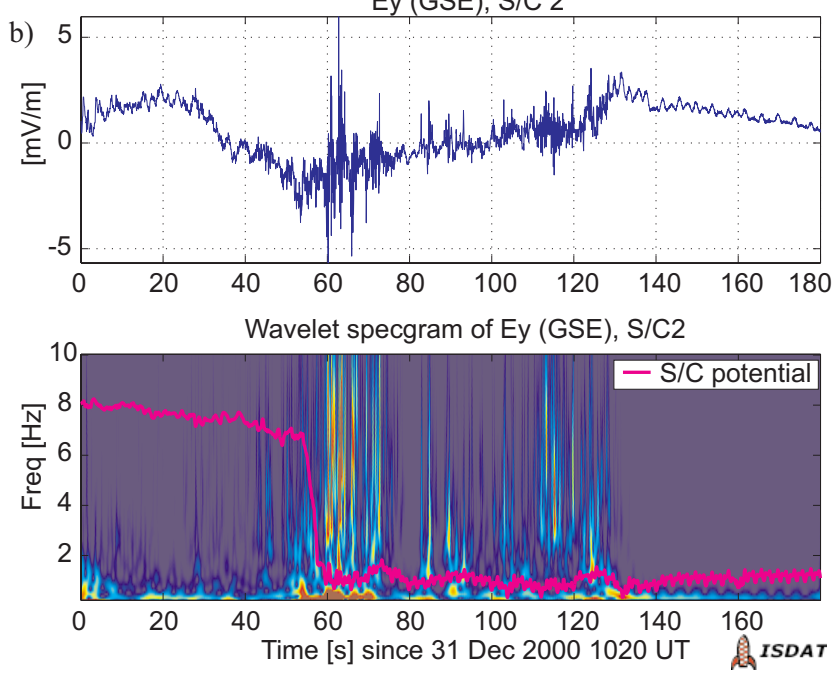

Fig. 6. (a) $E_{y}$ component of the electric field from satellite 2 (upper panel) and a wavelet transform of this signal (lower panel), using a linear color scale, for the boundary crossing leaving the magnetosphere. The potential $v_{p s}$ is indicated, see also panel a of Fig. 3, (b) similar to a, but for the boundary crossing entering the magnetosphere.

topause frame.

Our interpretation is that the electric field structures observed during the outbound crossing are rather static in the magnetopause frame, and move across the satellites with a velocity of about $100 \mathrm{~km} / \mathrm{s}$. The time scale of the structure is a few times $0.1 \mathrm{~s}$ in the satellite frame, corresponding to a length scale in the direction normal to the magnetopause of a few times 10 kilometers (as observed by single satellites). The structures are reasonably static during at least a few seconds (as observed by satellites crossing the magnetopause at different times). Along the magnetopause, the length scale is at least a few hundred kilometers (the separation between satellites).

In Fig. 8a, $v_{p s}$ for the inbound magnetopause crossing near 10:21 UT is shown for all four satellites. In Fig. 8b, 

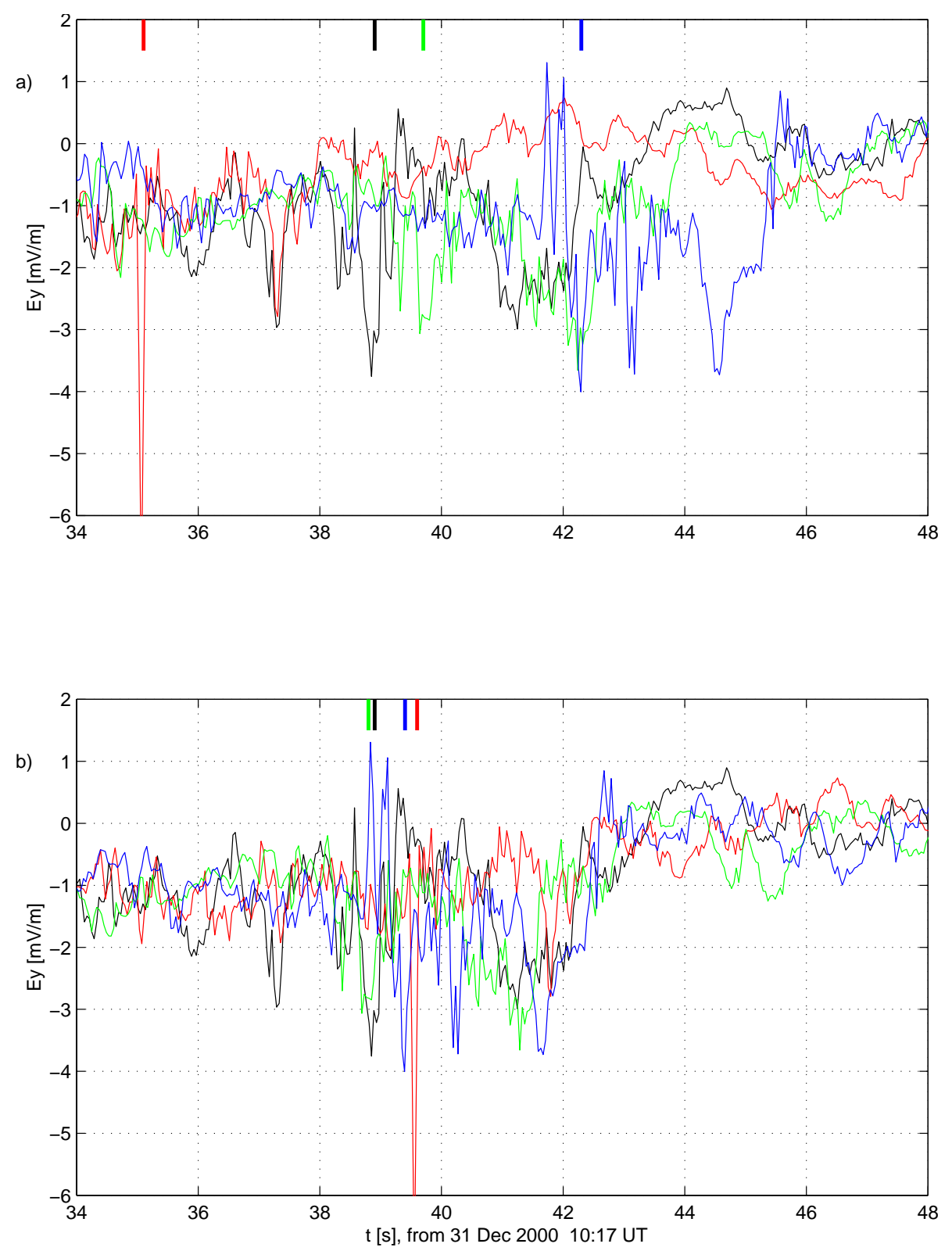

Fig. 7. (a) The $E_{y}$ component of the electric field from 10:17:00 UT on 31 Decemeber 2000, for all four Cluster satellites (1:black; 2:red; 3: green; 4:blue), (b) The same signals, but shifted in time to correspond to a common moving magnetopause frame.

these time series have been shifted, using an estimated magnetopause velocity of $(10,43,27) \mathrm{km} / \mathrm{s}$ in GSE coordinates. This corresponds to a speed of about $50 \mathrm{~km} / \mathrm{s}$. Compared to the outbound crossing, it is here less clear how to estimate the magnetopause speed. The blue line corresponding to satellite 4 indicates either a more structured magnetopause, or fast time variations. Using the estimated magnetopause velocity above, the $E_{y}$ components have been shifted in time and plotted in Fig. 8c. The red line corresponding to satellite 2 is the same electric field as in Fig. 6b. As for satellite 2, most enhancements of the electric field occur within about 10 seconds after the sharp decrease in $v_{p s}$. Satellites 1 and 2 show a couple of wavepackets with a few oscillations at a few $\mathrm{Hz}$ each. Satellites 3 and 4 show no clear wave packets, but changes in the electric field up to a few $\mathrm{mV} / \mathrm{m}$ can be found. No clear correlation between different satellites can be found.

Our interpretation is that the electric field structures observed during the outbound crossing are primarily due to time variations in the magnetopause frame. If static structures are present, their length scale along the magnetopause must be less than a few hundred kilometers (the satellite separation). Alternatively, they must last less than a few seconds (since they are not observed by satellites crossing the magnetopause at different times) and we would not call them "static". The time varying structures have a duration of a few seconds in the satellite frame, corresponding to a few hundred kilometers in the direction normal to the magnetopause 
(as observed by single satellites). The structures must last less than a few seconds (since they are not observed by satellites crossing the magnetopause at different times) or have a scale length along the magnetopause of less than a few hundred kilometers (the separation between satellites). Our discussion above only gives the limits for possible time and spatial variations. We interpret the structures as primarily time variations, since these limits exclude many reasonable essentially spatial variations, including very low frequency Alfvén waves with long wavelengths.

Figures 6, 7 and 8 show that the strongest electric fields occur on time scales of about a second, corresponding to a length scale of about one hundred kilometers in the frame of the moving magnetopause. This scale is comparable to an ion gyroradius. (A proton gyrofrequency of $0.3 \mathrm{~Hz}$ and a proton temperature of $100 \mathrm{eV}$ or $1 \mathrm{keV}$ correspond to gyroradii of about 100 or $300 \mathrm{~km}$, respectively).

It is interesting to put the details of the two magnetopause crossings discussed above into a larger perspective. The velocity of the magnetopause during the outbound crossing leaving the magnetosphere was $(-100,-10,-55) \mathrm{km} / \mathrm{s}$ in GSE coordinates and for the inbound crossing, (10, 43, 27) $\mathrm{km} / \mathrm{s}$. Since the satellites were positioned near the nominal duskside magnetopause, the magnetopause velocities are consistent with a depression wave, i.e. an inward bulge of magnetosheath plasma, moving past along the nominal magnetopause boundary. In this interpretation, the outbound crossing with static electric field structures corresponds to a large-scale wave reaching the spacecraft, while the inbound crossing with time varying electric field wave packets corresponds to a more turbulent "backside" of the large-scale wave.

The differences between the front side and the backside of large-scale magnetopause waves are known from other studies, (e.g. Rezeau and Russell, 1992; Bale et al., 2001). Preliminary investigations of other Cluster boundary crossings during the time period in Fig. 1 show both electric field single peaks and wavepackets. The strongest electric fields are typically around $5 \mathrm{mV} / \mathrm{m}$. These strong fields are found either just before the increase in $v_{p s}$, or just after the decrease in $v_{p s}$, as in Fig. 6, or a few seconds (typically a few hundred $\mathrm{km}$ ) into the magnetosphere, or they are co-located with the strongest gradient of $v_{p s}$. It might be that a rather flat front side of a large-scale wave is usually associated with a more static small-scale electric field, while a more turbulent backside of the large-scale wave is associated with smallscale time varying electric field packets. A statistical study is needed to confirm any differences in the fine structure of the electric field observed at the front and backsides of largescale magnetopause waves.

Preliminary investigations of the detailed STAFF magnetometer data show that the electric field structures in Figs. 6, 7 and 8 are accompanied by similar magnetic variations, either single peaks or wave packets. Before very detailed comparisons of the electric and magnetic fields are performed, it is useful to consider the average properties of the different fields.
One way to investigate the field oscillations is to study the ratio of the electric $E$ and magnetic $B$ power spectra. This ratio will of course vary near boundaries such as those in Fig. 3 since either $E$ or $B$ or both simultaneously, are large. Electric field power spectra from the boundary where satellite 4 is leaving the magnetosphere near 10:17:30 UT, are shown in Fig. 9a. The first (blue) spectrum corresponds to the region with the strongest electric fields, while the next (green) spectrum is from the region with somewhat weaker electric but stronger magnetic fields. The ratio $E / B$ is much smaller in the second case, as shown in Fig. 9b. Note that these figures give an average description of the boundary region, and do not indicate phenomena such as small-scale bursts of the electric field. Although the ratio $E / B$ changes by about a factor of 10 , the electric and magnetic perturbations could be due to same phenomenon if the change in the ratio is due to a change in background plasma parameters. Although the major field perturbations may be rather static in the magnetopause frame, in a preliminary investigation, it is still meaningful to investigate these fields in terms of the Alfvén velocity. We note that the quasi-static magnetic field magnitude is nearly constant, while the density changes by about a factor of $25\left(0.8\right.$ to $\left.20 \mathrm{~cm}^{-3}\right)$ during the boundary crossing near 10:17:30 UT, corresponding to a change in the Alfvén velocity by a factor of 5 . The ratio $E / B$ changes by a factor of about 10 during this crossing. In order for a comparison of $E / B$ at frequencies much higher than the proton gyrofrequency (about $0.3 \mathrm{~Hz}$ ) with the Alfvén velocity to be meaningful, some model must be used. A possible interpretation of broadband spectra in terms of Doppler shifted low frequency Alfvén waves is discussed by Stasiewicz et al. (2001), for other interpretations and discussions, see (Wahlund et al., 1998; Stasiewicz et al., 2000; Lund, 2001; Stasiewicz and Khotyaintsev, 2001). Electric field power spectra when satellite 4 is entering the magnetosphere near 10:21:00 UT are shown in Fig. 9c, together with the corresponding $E / B$ ratios in Fig. 9d. During this boundary crossing, the electric field is strong on the magnetospheric side of the boundary (blue line), but also during the decrease in $v_{p s}$ (green line) when the magnetic oscillations are also strong. As for the previous crossing, these figures give an average description of the boundary region. While Fig. 6 shows that phenomena occurring during a few seconds may dominate the electric field near the magnetopause, Fig. 9 shows that even when averaging over several seconds, great care must be used when selecting regions for detailed studies and that varying background plasma parameters must be considered.

\section{Discussion and Conclusions}

Broadband (in the satellite frame) electric field oscillations are common near the crossings of the boundary layer and the magnetopause, which is consistent with earlier observations, and these oscillations can have amplitudes at least up to $5 \mathrm{mV} / \mathrm{m}$. During a crossing, these waves are similar on all four Cluster satellites, i.e. they are likely to be distributed 

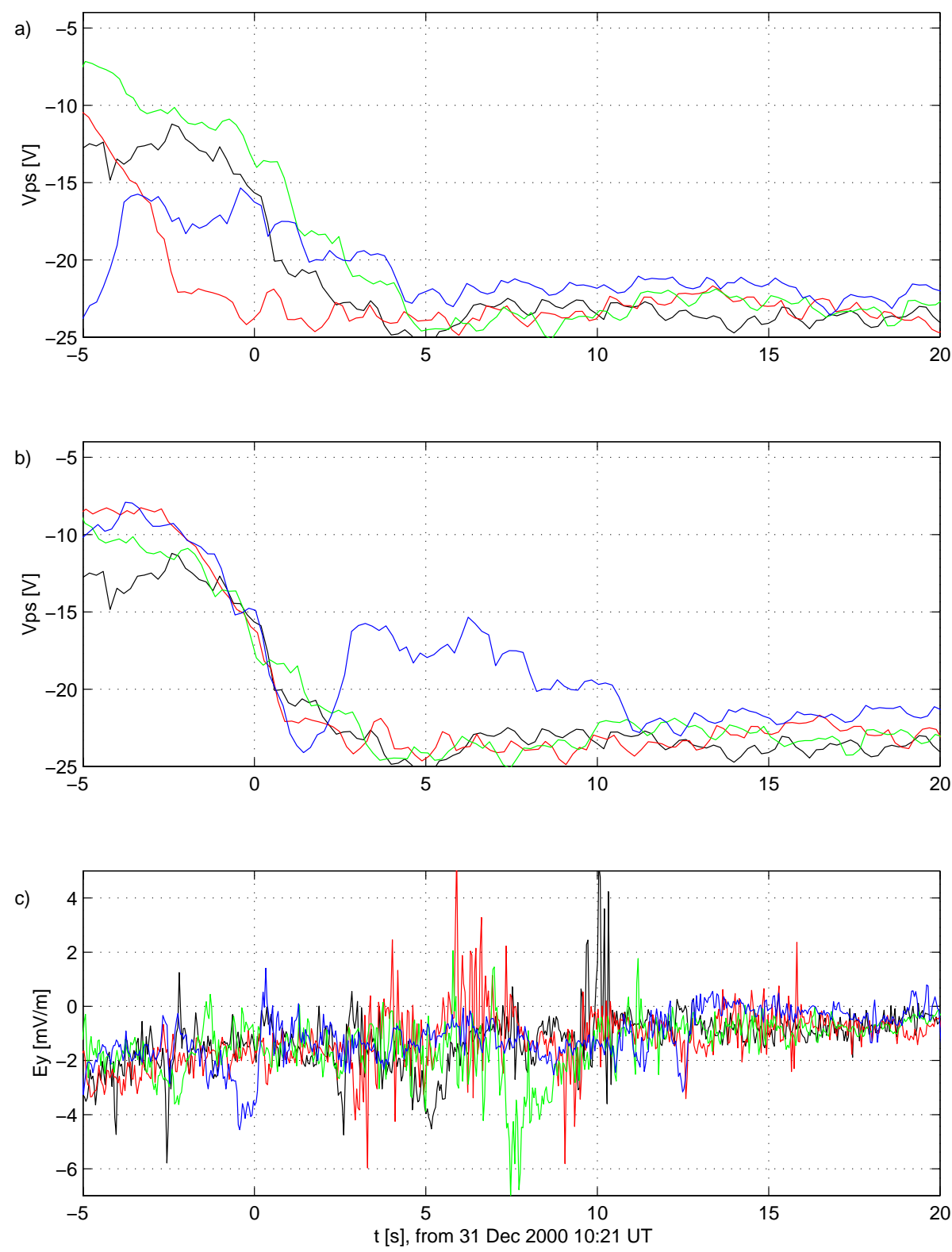

Fig. 8. (a) The potential $v_{p s}$ from 10:21:00 UT on 31 Decemeber 2000, for all four Cluster satellites (1: black; 2: red; 3: green; 4: blue), (b) The same signals, but shifted in time to correspond to a common moving magnetopause frame. (c) The $E_{y}$ component of the electric field for all four satellites, shifted as in panel (b).

over large areas of the boundary. We have studied waves up to $10 \mathrm{~Hz}$, corresponding to the approximate value of the local lower hybrid frequency. These oscillations typically occur near the Earthward edge of the boundary layer. Magnetic oscillations in the same frequency range are typically found in the boundary layer and across the magnetopause. The strongest electric fields occur during a few seconds, i.e. over distances of more than a few hundred $\mathrm{km}$ in the frame of the moving magnetopause, a scale length comparable to the ion gyroradius. Preliminary results indicate that on the front side of a large-scale magnetopause wave, the electric fields are rather static in the magnetopause frame, while the electric fields on the more turbulent "backside" of the large-scale wave consist of time varying electric field wave packets.

When investigating average wave properties over larger distances, corresponding to several seconds, great care must be taken since these properties may vary considerably near a boundary. It should be further investigated if changes in the average wave properties, such as the $E / B$ ratio, can be explained by varying background plasma parameters, together with some appropriate model.

Waves at the magnetopause can be important for transport across this boundary. To investigate the importance of the observed field oscillations, it is necessary to reveal their nature. Several possibilities should still be considered. Waves and nearly static structures can be generated by currents, density 

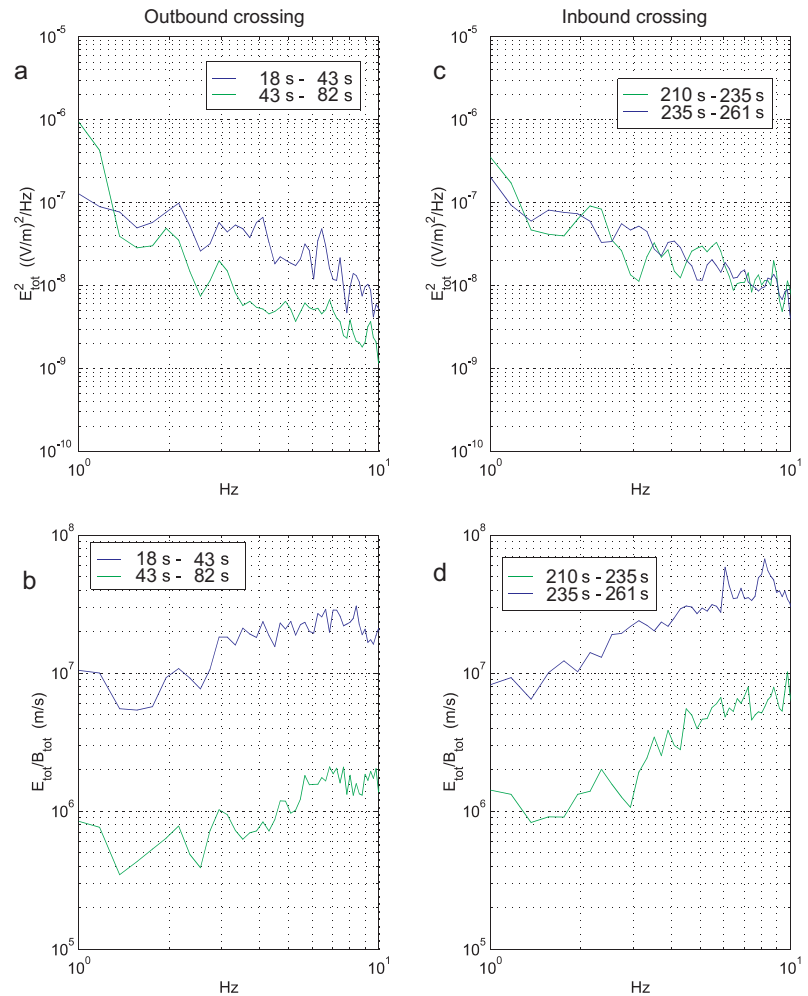

Fig. 9. (a) and (b) Electric field power spectra and $E / B$ ratios obtained when satellite 4 is leaving the magnetosphere, and (c) and (d) corresponding spectra when the satellite is entering the magnetosphere. The blue line correspond to the Earthward part of each event. Times are in seconds after 10:17:00 UT, 31 Dec 2000. The boundary between spectra for each crossing (43 $\mathrm{s}$ and $235 \mathrm{~s}$ ) are indicated in Fig. 3. The electric field $E$ is the total field in the satellite spin plane, while $B$ is the total magnetic field.

gradients, shear in the plasma flow, and possibly by other gradients. Waves may also propagate from other regions and accumulate at the magnetopause. In addition, very low frequency waves and static structures in the moving magnetopause frame of reference may occur at higher frequencies in the satellite frame. Although the electric wave fields are closely associated with the steep slope of $v_{p s}$, i.e. with a large relative change in density, generation by nearby currents and a changing $E / B$ ratio due to changing background parameters should still be considered. Currents at the magnetopause could, at least in principle, drive the lower hybrid drift instability (Winske et al., 1990; Shapiro et al., 1994; Drake et al., 1994). As in previous investigations, the density gradient is on the Earthward edge of the magnetopause (Eastman et al., 1996). Lower hybrid wave generation by density gradients, and its consequences for the magnetopause, has been considered by Gary and Sgro (1990), Treumann et al. (1991) and Lakhina et al. (1993). Doppler shift of nearly static structures to several $\mathrm{Hz}$ due to the motion of the magnetopause has been considered by Stasiewicz et al. (2001). In some scenarios, large wave amplitudes and reconnection occur without any local instability (Belmont and
Rezeau, 2001). In addition, shear in the plasma flow usually occurs across the magnetopause. One type of generation mechanism causing broadband waves at frequencies of the order of the ion gyrofrequency depends on shear due to inhomogeneous plasma flow, either caused by changes in the flow parallel to the ambient magnetic field (Gavrishchaka et al., 1999) or by inhomogeneous perpendicular flows (Amatucci, 1999; Koepke et al., 1999; Peñano and Ganguli, 2000). Recent investigations of Freja data show that such perpendicular shear may well contribute to the generation of waves at altitudes around $2000 \mathrm{~km}$ in the auroral region (Hamrin et al., 2001).

The EFW high resolution electric field observations on Cluster can be used to study the details of the electric field and wave structures near the magnetopause. In this article, we have used the probe-to-spacecraft potential $v_{p s}$ obtained on all four Cluster satellites to find density gradients, and to estimate the velocity of the magnetopause. This velocity is then used to study the electric field in the magnetopause frame. The magnetopause velocity during an event studied in detail is consistent with a depression wave, i.e. an inward bulge of the magnetosheath plasma, moving tailward along the nominal magnetopause boundary. Preliminary investigations indicate that a rather flat front side of the large-scale wave is associated with a rather static small-scale electric field, while a more turbulent backside of the large-scale wave is associated with small-scale time varying electric field wave packets. A statistical study is needed to confirm any consistent differences in the fine structure of the electric field observed at the front and backsides of large-scale magnetopause waves.

Acknowledgements. Contributions to the EFW instrument hardware and software by P. Harvey, G. Holmgren, A. Lundgren, B. Lybekk, K. Yearby and L. Åhlén are acknowledged.

Topical Editor M. Lester thanks P. Gough and another referee for their help in evaluating this paper.

\section{References}

Amatucci, W. E.: Inhomogeneous plasma flows, A review of in situ observations and laboratory experiments, J. Geophys. Res., 104, 14 481-14 504, 1999.

Anderson, B. J.: ULF signals observed near the magnetopause, in Physics of the magnetopause, (Eds) Song, P., Sonnerup, B. U. Ö., and Thomsen, M. F. pp. 269-276, AGU, Washington, 1995.

Bale, S.-D., Mozer, F.-S., André, M., Eriksson, A.-I., Gustafsson, G., Holmgren, G., Lindqvist, P.-A., Holtet, J., Lybekk, B., Pedersen, A., Grard, R., Laakso, H., Mursula, K., Tanskanen, P., and Yerby, K.: The normal, thickness and speed of the dusk magnetopause from Cluster Electric Field and Wave experiment measurements, Geophys. Res. Lett, submitted, 2001.

Balogh, A., Dunlop, M. W., Cowley, S. W. H., Southwood, D. J., Thomlinson, J. G., Glassmeier, K. H., Musmann, G., Lühr, H., Buchert, S., Acuña, M. H., Fairfield, D. H., Slavin, J. A., Riedler, W., Schwingenschuh, K., and Kivelson, M. G.: The Cluster Magnetic Field Investigation, Space Sci. Rev., 79, 65-91, 1997. 
Belmont, G. and Rezeau, L.: Magnetopause reconnection induced by magnetosheath Hall-MHD fluctuations, J. Geophys. Res., 105, accepted for publication, 2001.

Blecki, J., Kossaki, K., Popielawska, B., Klimov, S. I., Romanov, S. A., Savin, S. P., and Zeleny, L. M.: ELF plasma waves associated with plasma jets near the Earth magnetopause as observed by Prognoz-8, Phys. Scrip., 37, 623-631, 1988.

Cornilleau-Wehrlin, N., Chauveau, P., Louis, S., Meyer, A., Nappa, J. M., Perraut, S., Rezeau, L., Robert, P., Roux, A., de Villedary, C., de Conchy, Y., Friel, L., Harvey, C. C., Hubert, D., Lacombe, C., Manning, R., Wouters, F., Lefeuvre, F., Parrot, M., Pinçon, J. L., Poirier, B., Kofman, W., and Louarn, P.: The Cluster Spatio-Temporal Analysis of Field Fluctuations (STAFF) Experiment, Space Sci. Rev., 79, 107-136, 1997.

Drake, J. F., Gerber, J., and Kleva, R. G.: Turbulence and transport in the magnetopause current layer, J. Geophys. Res., 99, $11211-$ $11224,1994$.

Eastman, T. E., Fuselier, S. A., and Gosling, J. T.: Magnetopause crossings without a boundary layer, J. Geophys. Res., 101, 4957, 1996.

Escoubet, C. P., Schmidt, R., and Goldstein, M. L.: Cluster: Science and mission overview, Space Sci. Rev., 79, 11-32, 1997.

Fairfield, D. H.: Observations of the shape and location of the magnetopause: A review, in Physics of the magnetopause, (Eds) Song, P., Sonnerup, B. U. Ö., and Thomsen, M. F., pp. 53-60, AGU, Washington, 1995.

Gary, P. S. and Sgro, A. G.: The lower hybrid drift instability at the magnetopause, Geophys. Res. Lett., 17, 909-912, 1990.

Gavrishchaka, V. V., Ganguli, S. B., and Ganguli, G. I., Electrostatic oscillations due to filamentary structures in the magnetic-fieldaligned flow: The ion-acoustic branch, J. Geophys. Res., 104, 12683-12694, 1999.

Gurnett, D. A., Anderson, R. R., Tsurutani, B. T., Smith, E. J., Paschmann, G., Haerendel, G., Bame, S. J., and Russell, C. T.: Plasma wave turbulence at the magnetopause: Observations from ISEE 1 and 2, J. Geophys. Res., 84, 7043-7058, 1979.

Gustafsson, G., Boström, R., Holback, B., Holmgren, G., Lundgren, A., Stasiewicz, K., Åhlén, L., Mozer, F. S., Pankow, D., Harvey, P., Berg, P., Ulrich, R., Pedersen, A., Schmidt, R., Butler, A., Fransen, A. W. C., Klinge, D., Thomsen, M., Fälthammar, C.G., Lindqvist, P.-A., Christenson, S., Holtet, J., Lybekk, B., Sten, T. A., Tanskanen, P., Lappalainen, K., and Wygant, J.: The Electric Field and Wave Experiment for the Cluster Mission, Space Sci. Rev., 79, 137-156, 1997.

Gustafsson, G., André, M., Carozzi, T., Eriksson, A.-I., Fälthammar, C.-G., Grard, R., Holmgren, G., Holtet, J.-A., Ivchenko, N., Karlsson, T., Khotyaintsev, Y., Klimov, S., Laakso, H., Lindqvist, P.-A., Lybekk, B., Marklund, G., Mozer, F., Mursula, K., Pedersen, A., Popielawska, B., Savin, S., Stasiewicz, K., Tanskanen, P., Vaivads, A., and Wahlund, J.-E.: First results of electric field and density observations by Cluster EFW based on initial months of operation, Ann. Geophysicae, this issue, 2001.

Hamrin, M., André, M., Ganguli, G., Gavrishchaka, V. V., Koepke, M. E., Zintl, M. W., Ivchenko, N., Karlsson, T., and Clemmons, J. H.: Inhomogeneous transverse electric fields and wave generation in the auroral region: A statistical study, J. Geophys. Res., in press, 2001.

Holzer, R. E., McLeod, M. G., and Smith, E. J.: Preliminary results from the OGO 1 search coil magnetometer: Boundary positions and magnetic noise spectra, J. Geophys. Res., 71, 1481-1486, 1966.

Johnstone, A. D., Alsop, C., Burge, S., Carter, P. J., Coates, A. J.,
Coker, A. J., Fazakerley, A. N., Grande, M., Gowen, R. A., Gurgiolo, C., Hancock, B. K., Narheim, B., Preece, A., Sheather, P. H., Winningham, J. D., and Woodliffe, R. D.: Peace: A Plasma Electron and Current Experiment, Space Sci. Rev., 79, 351-398, 1997.

Koepke, M. E., Carroll, J. J., and Zintl, M. W.: Laboratory simulation of broadband ELF waves in the auroral ionosphere, J. Geophys. Res., 104, 14 397-14 416, 1999.

Labelle, J. and Treumann, R. A.: Plasma waves at the dayside magnetopause, Space Sci. Rev., 47, 175-202, 1988.

Lakhina, G. S., Shukla, P. K., and Stenflo, L.: Ultralow-frequency fluctuations at the magnetopause, Geophys. Res. Lett., 20, 24192422, 1993.

Lucek, E. A., Cargill, P., Dunlop, M. W., Kistler, L. M., Balogh, A., Baumjohann, W., Fornacon, K.-H., Georgescu, E., and Haerendel, G.: The magnetopause at high time resolution: Structure and lower-hybrid waves, Geophys. Res. Lett., 28, 681-684, 2001.

Lund, E. J.: Comment on "Identification of widespraed turbulence of dispersive Alfvén waves", Geophys. Res. Lett., 105, accepted for publication, 2001.

Peñano, J. R. and Ganguli, G.: Generation of ELF electromagnetic waves in the ionosphere by localized transverse dc electric fields: Subcyclotron frequency regime, J. Geophys. Res., 105, 74417458, 2000.

Pedersen, A.: Solar wind and magnetosphere plasma diagnostics by spacecraft electrostatic potential measurements, Ann. Geophysicae, 13, 118-129, 1995.

Pedersen, A., Décréau, P., Escoubet, C. P., et al.: Cluster four-point high time resolution information on electron densities, Ann. Geophysicae, this issue, 2001.

Rezeau, L., A. R. and Russell, C. T.: Can ULF fluctuations observed at the magnetopause play a role in anomalous diffusion?, Proceedings of the 26th ESLAB Symposium, ESA SP-346, 346, 127-131, 1992.

Rezeau, L. and Belmont, G.: Magnetic turbulence at the magnetopause, a key problem for understanding the solar wind/magnetosphere exchanges, Space Sci. Rev., 95, 427-441, 2001.

Rezeau, L., Roux, A., and Russell, C. T.: Characterization of smallscale structures at the magnetopause from ISEE measurements, J. Geophys. Res., 98, 179-186, 1993.

Russell, C. T.: The structure of the magnetopause, in Physics of the magnetopause, (Eds) Song, P., Sonnerup, B. U. Ö., and Thomsen, M. F., pp. 81-98, AGU, Washington, 1995.

Shapiro, V. D., Shevchenko, V. I., Cargill, P. J., and Papadopoulos, K.: Modulational instability of lower hybrid waves at the magnetopause, J. Geophys. Res., 99, 23 735-23 740, 1994.

Sibeck, D. G., Paschmann, G., Treumann, R. A., Fuselier, S. A., Lennartsson, W., Lockwood, M., Lundin, R., Ogilvie, K. W., Onsager, T. G., Phan, T.-D., Roth, M., Scholer, M., Sckopke, N., Stasiewicz, K., and Yamauchi, M.: Plasma transfer processes at the magnetopause, in Magnetospheric plasma sources and losses, (Eds) Hultqvist, B., Øieroset, M., Paschmann, G., and Treumann, R., pp. 207-283, Kluwer Academic Publishers, Dordrecht, 1999.

Stasiewicz, K. and Khotyaintsev, Y.: Reply to comment on "Identification of widespraed turbulence of dispersive Alfvén waves, Geophys. Res. Lett., 105, accepted for publication, 2001.

Stasiewicz, K., Khotyaintsev, Y., Berthomier, M., and Wahlund, J.E.: Identification of widespraed turbulence of dispersive Alfvén waves, Geophys. Res. Lett., 27, 173-176, 2000.

Stasiewicz, K., Khotyaintsev, Y., Gustafsson, G., and Eriksson, A. I.: Drift-Kinetic Alfvén waves observed by Cluster at the mag- 
netopause, Ann. Geophysicae, submitted, 2001.

Thorne, R. M. and Tsurutani, B. T.: Wave-particle interactions in the magnetopause boundary layer, in Physics of Space Plasmas, (Eds) Chang, T., Crew, G. B., and Jasperse, J. R., pp. 119-150, Scientific Publishers, Cambridge, 1990.

Treumann, R. A., Labelle, J., and Pottelette, R.: Plasma diffusion at the magnetopause: The case of lower hybrid drift waves, J. Geophys. Res., 96, 16 009-16013, 1991.

Tsurutani, B. T., Brinca, A. L., Smith, E. J., Okida, R. T., Anderson, R. R., and Eastman, T. E.: A statistical study of ELF-VLF plasma waves at the magnetopause, J. Geophys. Res., 94, 1270-1280,
1989.

Wahlund, J.-E., Eriksson, A.-I., Holback, B., Boehm, M.-H., Bonnell, J., Kintner, P.-M., Seyler, C.-E., Clemmons, J.-H., Eliasson, L., Knudsen, D.-J., Norqvist, P., and Zanetti, L.-J.: Broadband ELF plasma emissions during auroral energization, 1. Slow ion acoustic waves, J. Geophys. Res., 103, 4343-4375, 1998.

Winske, D., Gary, S. P., and Lemons, D. S.: Diffusive transport at the magnetopause, in Physics of Space Plasmas, (Eds) Chang, T., Crew,G. B., and Jasperse, J. R., pp. 397-415, Scientific Publishers, Cambridge, 1990. 